\title{
Retropelvic artery: anatomical background for experimental urology (Review article)
}

\author{
Marco Aurélio Pereira-Sampaio,,2 Diogo Benchimol de Souza, ${ }^{1}$ Francisco José Sampaio' \\ 'Urogenital Research Unit, State University of Rio de Janeiro, Rio de Janeiro, RJ, Brazil \\ 2Department of Morphology, Fluminense Federal University, Niterói, RJ, Brazil
}

Disclose and conflicts of interest: none to be declared by all authors

\begin{abstract}
Introduction: transoperative and postoperative bleeding still are important complications in urologic procedures at the upper pole. A detailed review of the retropelvic artery anatomy would be helpful to surgeons. The aim of the present study was to revisit the anatomy of the retropelvic artery and its importance for urology, as well as evaluate the presence and morphology of this artery in animal models. The posterior division of the renal artery (retropelvic artery) describes an arch close to the upper infundibulum (57\%) or crosses the posterior aspect of the renal pelvis $(43 \%)$. Simulated partial nephrectomy of the upper pole shows that partial nephrectomies performed at less than $1.0 \mathrm{~cm}$ of the hilar superior margin impair the retropelvic artery. When the retropelvic artery is damaged, it could cause destruction of a large area of the remaining renal posterior parenchyma, sometimes leading to postoperative leak. Although retropelvic artery is not present in any animal model on the same way as in humans, when simulated partial nephrectomy is performed in the cranial pole of pig kidneys, the outcomes are quite similar to the same procedure carried out in humans. So, even though the retropelvic artery was not demonstrated in any animal model, it is not possible to state that sections of the cranial pole in those animals would injury important arteries for the kidney parenchyma, as in humans and pigs.
\end{abstract}

Keywords: Retropelvic artery, Kidney hemorrhage, Partial nephrectomy,Intrarenal access, Animal model.

\section{Introduction}

The kidney anatomy has been studied along the centuries. However, only in 1873, the first important description of the intrarenal arteries of the kidney showed that the renal pelvis of the antelope gnu separates two arterial segments, one ventral and other dorsal. ${ }^{1}$ Later, Brodel $^{2}$ reported the general branching pattern of the human renal artery, demonstrating the lack of anastomosis and the avascular line between the anterior and posterior segments on the posterior area of the lateral margin of the kidney. The knowledge of the Brodel's avascular line is still useful for several procedures, including new treatment modalities, such as robot-assisted anatrophic nephrolithotomy. ${ }^{3}$ Then, the intrarenal arterial distribution was described in details, and the renal parenchyma was divided into specific and reasonably constant anatomical segments. ${ }^{4}$ So, the kidney was divided into 5 arterial segments: apical, upper (anterior), middle (anterior), lower and posterior. Others also studied the intrarenal arteries and their surgical relevance. ${ }^{5-8}$ Finally, Sampaio and Aragão ${ }^{9}$ carried out a systematic study demonstrating the anatomical relationship between the intrarenal arteries and the kidney collecting system, and reporting much useful information for the surgical setting. This anatomical study in tridimensional endocasts stimulated more investigation on the important aspects of the kidney, which could improve the usual practices of urologic procedures, such as ureteropelvic junction (UPJ) access, ${ }^{10-12}$ intrarenal access ${ }^{13-15}$ and nephron sparing surgery ${ }^{16}$ The kidney arterial segments were proportionally evaluated, demonstrating that the posterior segment is constant and supplied by the posterior segmental artery, often known as the retropelvic artery. This segment had the greatest mean value of proportional area and also the greatest maximum value of proportional area, evidencing the significance of the retropelvic artery. ${ }^{17}$

The use of new imaging methodologies increased detection of incidental renal masses. ${ }^{18}$ More than 70,000 new renal cancers were estimated for 2015 and more than 14,000 deaths will be attributed to kidney cancer in the United States of America. ${ }^{19}$ These cases are mostly small solitary masses, with less than $4 \mathrm{~cm}$ of diameter, classified as T1a. ${ }^{20}$ The American Urological Association's evaluated the advantages of partial nephrectomy (PN) and recommends it as the gold standard therapy for all T1a cancers, and T1b (solitary masses $<7 \mathrm{~cm}$ ) cancers in many settings ${ }^{21}$ Retrospective and prospective studies showed that renal function after $\mathrm{PN}$ is better when compared to radical nephrectomy $(\mathrm{RN}){ }^{22,23}$ On the other hand, the risk for perioperative bleeding and urinary fistula was increased for PN if compared with $\mathrm{RN}^{24}$ The postoperative hemorrhage is a complication for both open partial nephrectomy 
$(\mathrm{OPN})$ and laparoscopic partial nephrectomy (LPN). ${ }^{25}$ One of the most common reasons for conversion PN to RN was the continued intraoperative bleeding after resection. ${ }^{26}$ The renal ischemia/reperfusion is widely used to avoid intraoperative bleeding, but the kidney injury is considered an important issue that may lead to renal failure. ${ }^{27,28}$ Several energy sources have been employed in LPN to minimize the level of difficulty of the procedure and blood loss without the need for hilar occlusion. ${ }^{29}$ The LPN was widespread adopted, and more recently the robot assisted partial nephrectomy (RAPN) has been studied extensively, in order to finding a more secure minimally invasive nephron sparing approach. ${ }^{30}$ Partial nephrectomy of the upper pole is more complex due to the pathway of the retropelvic artery, which cross the upper infundibulum and turn to de mid zone. Hence, the resection of the renal mass may damage the artery, leading to a loss of arterial supply at the mid zone and a postoperative urinary leak. ${ }^{9,16}$

The American Urological Association and Endourology Society Surgical Management of Stones Guidelines recommend percutaneous nephrolithotomy (PNL) as the gold standard therapy for intrarenal stones higher than $2 \mathrm{~cm} .{ }^{31}$ Laparoscopic pyelolithotomy (LP) reduces analgesic requirements, hospital stay, and blood loss. ${ }^{32}$ Venous bleeding during neprhostomy is usually resolved by clamping nephrostomy tube or administration of mannitol, but arterial bleeding is more worrying. ${ }^{33}$ The most significant complication of PNL is bleeding requiring transfusion, due to the high vascular density of the kidney. ${ }^{34}$ A lower incidence of postoperative bleeding was associated with the LP when compared to PNL. ${ }^{35-37}$ Robotic assisted surgery are also used to treat large staghorn stone, such as robotic pyelolithotomy (RP), robotic nephrolithotomy and robotic anatrophic nephrolithotomy (RAN), and the indications are the most diverse, including anatomic malformations that precludes a safe percutaneous nephrolithotomy and ureteropelvic junction obstruction. ${ }^{38}$

Since the transoperative and postoperative bleeding stillareimportantcomplicationsinurologic procedures, the knowledge of the detailed relationship between the retropelvic artery and the collecting system ${ }^{9,16}$ will be helpful to surgeons to perform procedures at the upper pole and the posterior surface, such as laparoscopic partial nephrectomy, pielolithotomy and nephrolithotomy, avoiding renal hemorrhage. The aim of the present study was to revisit the anatomy of the retropelvic artery and its importance for urology, as well as evaluate the presence and morphology of this artery in animal models.

\section{Anatomy of the Retropelvic Artery}

Primarily, when the renal artery is single, it splits into two main branches, one anterior and other posterior, which branch towards to the lateral border of the kidney. ${ }^{4}$ The anterior division supplies branches to the apical, upper, middle and inferior segments, while the posterior division give branches to posterior and inferior segments. ${ }^{9}$

The posterior division of the renal artery (retropelvic artery) has a variable pathway. Sampaio and Aragão, ${ }^{9}$ reported that retropelvic artery describes an arch after to get into the hilum, running quite close to the upper infundibulum or to the region between the upper infundibulum and the renal pelvis in $57 \%$ of the kidneys (Figure 1A). The simulated partial nephrectomy of the upper pole shows that partial nephrectomies performed at less than $1.0 \mathrm{~cm}$ of the hilar superior margin present damage to the retropelvic artery. ${ }^{39}$ Therefore, this artery is at great risk during nephron sparing surgery of the upper pole. For this reason, an imaging examination to know the exact pathway of the retropelvic artery is very important before resection of the superior pole, because injury to the retropelvic artery will result in significant hemorrhage. ${ }^{16}$ In the other usual pathway, the retropelvic artery crosses the posterior aspect of the renal pelvis in $43 \%$ of the kidneys (Figure 1B). It was demonstrated that direct dorsal puncture of the renal pelvis cause damage in retropelvic artery in $16.1 \%$ of cases, while caliceal fornix puncture only damage veins of the upper pole in $7.7 \%$ of cases ${ }^{14}$ Along its route, the retropelvic artery gives off interlobar branches to the upper pole, ${ }^{9}$ and often ends on the region between the lower pole and the renal pelvis (62\%), however, in the remaining cases (38\%), the retropelvic artery reaches the lower pole, sending interlobar branches to the posterior calyces of the inferior segment. ${ }^{9}$

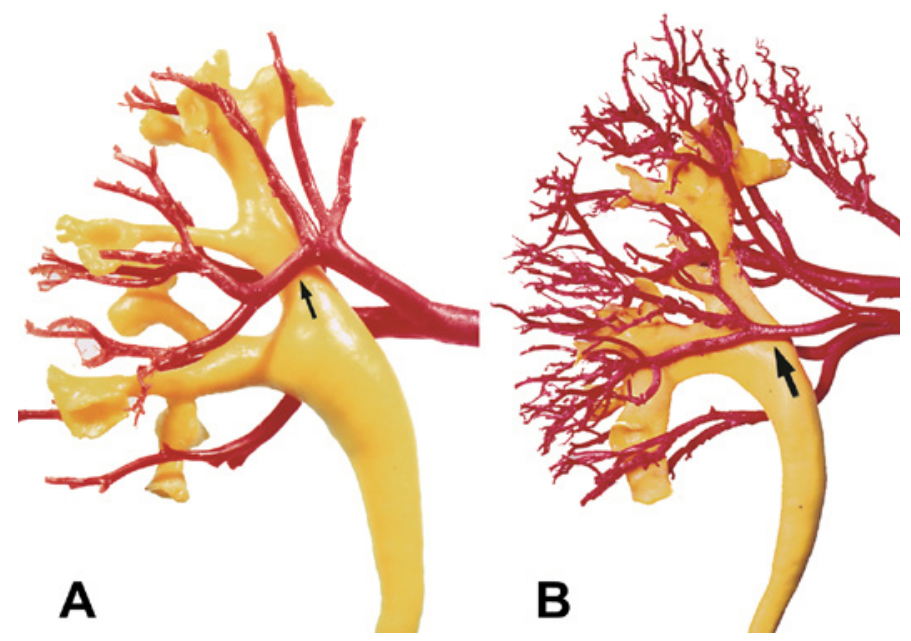

Figure 1. Posterior view of two endocasts of collecting system together with the intrarenal arteries from left human kidneys. A. The posterior division of the renal artery (retropelvic artery) describes an arch close to the region between the upper infundibulum and renal pelvis (arrow). B. The retropelvic artery crosses the posterior aspect of the renal pelvis (arrow). (Figure 1A was reprinted with permission from Sampaio $\mathrm{FJB}^{50}$ and Figure $1 \mathrm{~B}$ was reprinted with permission from Sampaio FJB46). 
Besides, when the retropelvic artery extent to the lower pole, it has a direct relationship with the dorsal surface of the ureteropelvic junction (UPJ) in $3.5 \%$ of the kidneys, or this artery crosses the renal pelvis at less than $1.5 \mathrm{~cm}$ above the posterior surface of the UPJ in $6.8 \%$ of cases. ${ }^{10}$ Therefore, posterior or posterolateral incisions at the UPJ must be avoided. In the UPJ stenosis, the deep incision along the stenotic UPJ wall should be made just at a lateral position. The incision performed at this nonvascular area of the UPJ, will avoid the retropelvic artery that can be related posteriorly to or less than $1.5 \mathrm{~cm}$ above the posterior surface of the UPJ.11,12,15

The posterior segmental artery (retropelvic artery) supplies the posterior segment, which is present in $100 \%$ of cases. This segment had the greatest median value of proportional area (33.76\%) and also the greatest maximum value of proportional area, accounting for to $52.93 \%$ of the total kidney area. ${ }^{17}$ When the retropelvic artery is damaged, the ligature to control bleeding could cause destruction of a large area of the remaining renal posterior parenchyma, sometimes leading to postoperative leak. ${ }^{16}$

\section{Retropelvic Artery In Animal Models}

Pigs have been used as model for kidney research and training due to the anatomical resemblance with human kidney. ${ }^{40}$ However, the intrarenal arteries do not branch exactly as the renal artery of the human kidney ${ }^{41}$ The main difference is the primary division of the renal artery, which form cranial and caudal branches (Figure 2), ${ }^{41}$ while in humans the renal artery primarily divides into an anterior and posterior branches. ${ }^{9,42}$ This difference in the primary branches of the renal artery also makes the arterial segmentation very different, because the segmentation is directly dependent on the extra hilar branching of the renal artery. ${ }^{17}$ Thereby, the main arterial segment in pigs is the cranial segment with a mean proportional area of $50 \%,{ }^{43}$ different from the human kidney, where the main segment is the posterior with a mean proportional area of $33.76 \% .{ }^{17}$ Although retropelvic artery is not present in pigs on the same way as in humans, cranial division of the renal artery gives off a dorsal branch in $47.25 \%$ of kidneys (Figure 2A), which crosses the dorsal surface of the renal pelvis, ${ }^{41}$ resembling the arrangement found in $43 \%$ of humans kidneys. ${ }^{9}$ The dorsal artery (retropelvic artery) from the cranial division of the renal artery of pigs has no relationship with the cranial infundibulum as occur in $57 \%$ of the human kidneys. ${ }^{9,41}$ Nevertheless, when the simulated partial nephrectomy was performed in the cranial pole of pig kidneys, the outcomes are quite similar to the same procedure carried out in humans. The retropelvic artery of the human kidney and the cranial division of the renal artery (before the origin of the dorsal branch) were damaged in transverse sections less than $1.0 \mathrm{~cm}$ from the hilar border. ${ }^{39,44} \mathrm{In}$ this cases, the dorsal hilar zone lost the arterial supply (Figure 2B), probably leading to a urinary fistula and urine leaking in a surgical setting.

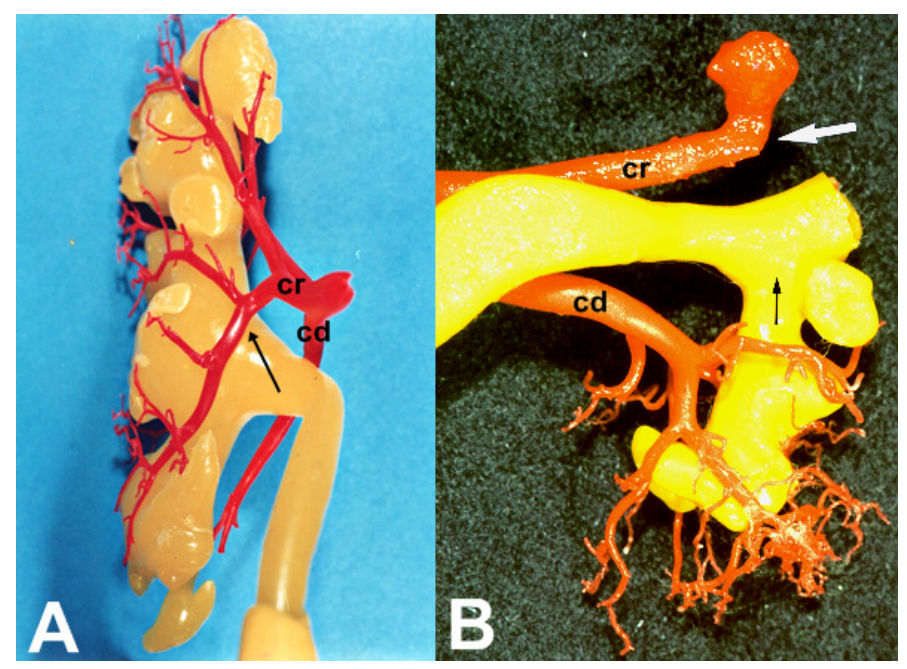

Figure 2. A. Dorsal view of an endocasts of collecting system together with the intrarenal arteries from a left pig kidney. Dorsal branch of the cranial division of the renal artery crosses the dorsal surface of the renal pelvis (arrow). B. Dorsal view of an endocasts of collecting system together with the intrarenal arteries from a right pig kidney, where simulated partial nephrectomy of the cranial pole was performed in the hilar margin. The cranial division of the renal artery was damaged (white arrow), leaving the dorsal hilar zone without arterial supply (black arrow). cr - cranial division of the renal artery; cd - caudal division of the renal artery.

As in the human kidney, intrarenal access in the pig kidney should be made close to the lateral border, through the caliceal fornix..$^{14}$ Punctures in the dorsal surface of the renal pelvis may result in a vascular complication, as in the human kidney, due to the course of the posterior branch from the cranial division of the renal artery, which crosses the dorsal surface of renal pelvis in $47.25 \%{ }^{41}$

The renal artery of the dog splits into dorsal and ventral branches. The dorsal division of the renal artery divides into cranial and caudal branches, which give off interlobar branches that course toward to the cortex in $60 \%$ of kidneys. ${ }^{45}$ In only $4.2 \%$ of kidneys, the dorsal hilar region is irrigated by interlobar branches from the cranial branch of the dorsal division of the renal artery. ${ }^{45}$ Although these branches are related to the renal pelvis, they are quite different from the retropelvic artery from human kidney (Figure 3A). Moreover, the dorsal surface of the UPJ in dogs always presented an important artery, originating from the caudal branch of the dorsal division of the renal artery. ${ }^{45}$ This is different from the human kidney, where the UPJ posterior surface is related to the retropelvic in only $3.5 \%{ }^{10}$

The renal artery of the rabbit divides into dorsal and ventral branches. In $68.29 \%$ of the kidneys, the dorsal branch originates three segmental arteries: cranial, 
middle and caudal. In the remaining kidneys, the dorsal branch divides into two segmental arteries: cranial and caudal $(24.39 \%)$ or middle and caudal $(7.32 \%)$. In all these cases, the dorsal hilar zone is irrigated by interlobar branches from the cranial segmental arteries in only $17 \%$ (Figure 3B). In addition, the course of them is almost horizontal, ${ }^{46}$ completely different from the retropelvic artery from human kidney. ${ }^{9}$ In rabbit, like in dogs, ${ }^{45}$ the dorsal surface of the UPJ is related to an important artery in all kidneys, ${ }^{46}$ also different from the human kidney. ${ }^{10}$ with the kidney collecting system. Even so, the branching pattern of the rat intrarenal arteries is very similar to that one found in dogs. ${ }^{48}$ Therefore, it is very likely that there is no retropelvic artery in rats, but more studies should be done to analyze the relationship between the intrarenal arteries and the collecting system in this specie. More recently, Szymanski and colleagues ${ }^{49}$ reported the branching pattern of the intrarenal arteries of the bovine, and its relationship with the collecting system. Although the manuscript did not mention the presence of a

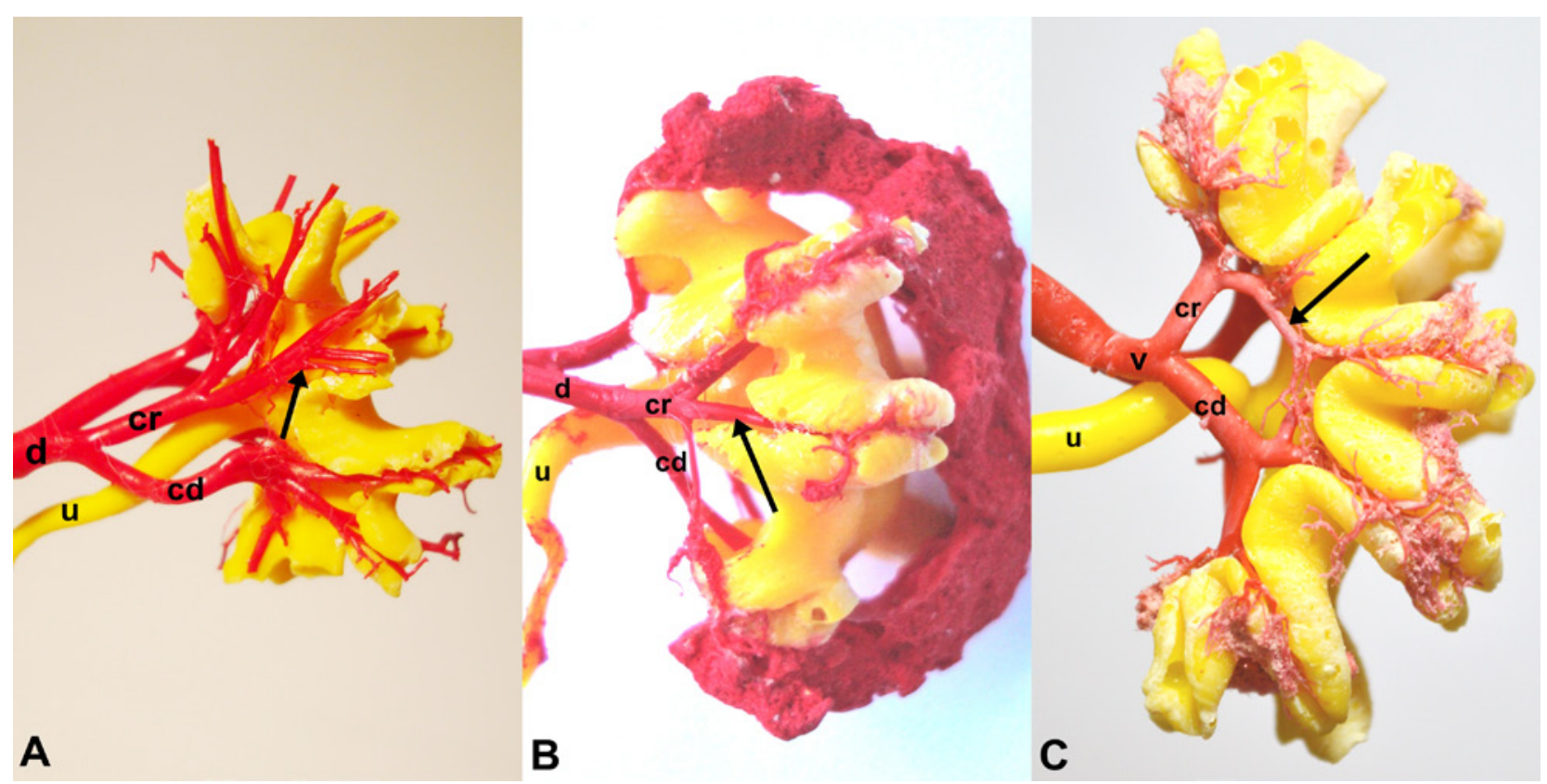

Figure 3. A. Dorsal view of an endocasts of collecting system together with the intrarenal arteries from a right dog kidney. The dorsal hilar region is irrigated by branches from the cranial branch of the dorsal division of the renal artery (arrow). B. Dorsal view of an endocasts of collecting system together with the intrarenal arteries from a right rabbit kidney. The dorsal hilar zone is irrigated by one branch from the cranial segmental artery with a horizontal course (arrow). C. Ventral view of an endocasts of collecting system together with the intrarenal arteries from a left sheep kidney. The cranial segmental artery of the ventral division of the renal artery gives off a branch to the ventral hilar zone with an oblique course (arrow). $u$ - ureter; $d$ - dorsal division of the renal artery; $v$ - ventral division of the renal artery; cr - cranial branch; cd - caudal branch. (Figure 3C was reprinted with permission from Buys-Gonçalves GF, et al. ${ }^{46}$ ).

The sheep kidney does also not have a retropelvic artery related to the upper infundibulum as humans. The cranial segmental artery of both dorsal and ventral divisions of the renal artery give off a branch to the dorsal and ventral hilar zones in $31,58 \%$ and $18.42 \%$ of the kidneys, respectively, ${ }^{47}$ In some cases, these branches course obliquely on the renal pelvis (Figure 3C), reminding the arterial supply of the dorsal hilar zone of the pig kidney. ${ }^{41}$ As it was demonstrated in pigs, this artery is not exactly like the retropelvic artery from the human kidney, but when transverse sections were made to simulate the partial nephrectomy of the cranial pole, it showed the same vascular injury. ${ }^{39,44}$ The UPJ is also related to an important artery in the dorsal surface in all casts. ${ }^{47}$

The intrarenal arteries of the rat were already described, but there is no report on its relationship retropelvic artery, in some figures it seems that this artery is present in this specie. So, a detailed study of the intrarenal anatomy in bovine kidney can evaluate if this animal could be a good model for nephron sparing surgery..$^{50}$

The proportional evaluation of the arterial segments was not studied in other animals than in pigs and humans. Likewise, simulated study of the partial nephrectomy of the cranial pole to assess the vascular injury was also not performed in other models, except in pigs. So, although the retropelvic artery was not demonstrated in any animal model, it is not possible to state that transverse sections of the cranial pole in those animals would injury important arteries for the kidney dorsal surface, as in humans and pigs. 


\section{References}

1. Hyrtl J. Corrosions Anatomie. Vienna: W Braumuller; 1873:220.

2. Brodel M. The intrinsic blood-vessels of the kidney and their significance in nephrotomy. Bull Johns Hopkins Hosp 1901;12:10-13.

3. King SA, Klaassen Z, Madi R. Robot-assisted anatrophic nephrolithotomy: description of technique and early results. J Endourol 2014;28(3):325-329.

4. Graves FT. The anatomy of the intrarenal arteries and its application to segmental resection of the kidney. Brit J Surg 1954;42:132-139.

5. Fine $H$, Keen EN. The arteries of the human kidney. J Anat 1966;100:881-894.

6. Hodson J. The lobar structure of the kidney. Brit J Urol 1972;44:246261.

7. Boyce WH. Nephrolithotomy. In: Glenn JF, eds. Urologic Surgery, 3rd ed. Philadelphia: JB Lippincott Co, 1983:181-194.

8. Graves FT. The anatomy and nomenclature of the intrarenal arteries. In: Graves FT, eds. Anatomical Studies for Renal and Intrarenal Surgery. Bristol: Wright, 1986:13-21.

9. Sampaio FJ, Aragão, AHBM. Anatomical relationship between the intrarenal arteries and the kidney collecting system. J Urol 1990;143:679-681.

10. Sampaio FJ. Relationship between segmental arteries and pelviureteric junction. Br J Urol 1991;68:214-217.

11. Sampaio FJ. The dillemma of the crossing vessel at UPJ. Precise anatomic study. J Endourol 1996;10:411-415.

12. Sampaio FJ. Vascular anatomy at the ureteropelvic junction. Urol Clin North Am 1998;25:251-258.

13. Sampaio FJ. Intrarenal endourologic surgery. Anatomical background. J Endourol 1992;6:301-304.

14. Sampaio FJ, Zanier JFC, Aragão AHM, Favorito LA. Intrarenal access: Three-dimensional anatomical study. J Urol 1992;148:1769-1773.

15. Sampaio FJ, Favorito LA. Ureteropelvic junction stenosis: vascular anatomical background for endopyelotomy. J Urol 1993;150:1787-1791. 16. Sampaio FJ. Anatomical background for nephron-sparing surgery in renal cell carcinoma. J Urol 1992;147:999-1005.

17. Sampaio FJ, Schiavini JL, Favorito LA. Proportional analysis of the kidney arterial segments. Urol Res 1993;21:371-374.

18. Hock LM, Lynch J, Balaji KC. Increasing incidence of all stages of kidney cancer in the last 2 decades in the United States: an analysis of surveillance, epidemiology and end results program data. J Urol 2002;167:57-60.

19. Siegel RL, Miller KD, Jemal A. Cancer statistics, 2020. CA Cancer J Clin 2020;70:7-30.

20. Russo P. Renal cell carcinoma: presentation, staging, and surgical treatment. Semin Oncol 2000;27:160-176.

21. Campbell SC, Novick AC, Belldegrun A, et al. Guideline for management of the clinical T1 renal mass. J Urol 2009;182:1271-1279. 22. Kim SP, Murad MH, Thompson RH, et al. Comparative Effectiveness for Survival and Renal Function of Partial and Radical Nephrectomy for Localized Renal Tumors: A Systematic Review and Meta-Analysis. J Urol 2012;188:51-57.

23. Scosyrev E, Messing EM, Sylvester R, Campbell S, Van Poppel $H$. Renal function after nephron-sparing surgery versus radical nephrectomy: results from EORTC randomized trial 30904. Eur Urol 2014;65:372-377.

24. Kunath F, Schmidt S, Krabbe LM, et al. Partial nephrectomy versus radical nephrectomy for clinical localised renal masses. Cochrane Database Syst Rev 2017;5:CD012045.

25. Lane BR, Novick AC, Babineau D, Fergany AF, Kaouk JH, Gill IS. Comparison of laparoscopic and open partial nephrectomy for tumor in a solitary kidney. J Urol 2008;179:847-851.

26. Petros FG, Keskin SK, Yu KJ, et al. Intraoperative conversion from partial to radical nephrectomy: incidence, predictive factors, and outcomes. Urology 2018;116:114-119.

27. Lee H, Song BD, Byun SS, Lee SE, Hong SK. Impact of warm ischemia time on postoperative renal function after partial nephrectomy for clinical T1 renal cell carcinoma: a propensity score-matched study. BJU Int 2018;121:46-52.

28. Zabell JR, Wu J, Suk-Ouichai C, Campbell SC. Renal Ischemia and Functional Outcomes Following Partial Nephrectomy. Urol Clin North
Am 2017;44:243-255.

29. Rubinstein M, Moinzadeh A, Colombo JR, Favorito LA, Sampaio FJ, Gill IS. Energy Sources for Laparoscopic Partial Nephrectomy -Critical Appraisal. International. Braz J Urol 2007;33:03-10.

30. Ghani KR, Sukumar S, Sammon JD, Rogers CG, Trinh QD, Menon $M$. Practice patterns and outcomes of open and minimally invasive partial nephrectomy since the introduction of robotic partial nephrectomy: results from the nationwide inpatient sample. J Urol 2014;191:907-912.

31. Assimos D, Krambeck A, Miller NL, et al. Surgical management of stones: American Urological Association/Endourological Society Guideline, PART I. J Urol 2016;196:1153-1160.

32. Singal R, Dhar S. Retroperitoneal laparoscopic pielolithotomy in renal pelvic stone versus open surgery - a comparative study. Clujul Med 2018;91:85-91.

33. Wollin DA, Preminger GM. Percutaneous nephrolithotomy: complications and how to deal with them. Urolithiasis 2018;46:87-97. 34. Michel MS, Trojan L, Rassweiler JJ. Complications in percutaneous nephrolithotomy. Eur Urol 2007;51:899-906.

35. Tefekli A, Tepeler A, Akman T et al: The comparison of laparoscopic pyelolithotomy and percutaneous nephrolithotomy in the treatment of solitary large renal pelvic stones. Urol Res 2012;40:549-555.

36. Al-Hunayan A, Khalil M, Hassabo $M$ et al: Management of solitary renal pelvic stone: laparoscopic retroperitoneal pyelolithotomy versus percutaneous nephrolithotomy. J Endourol 2011;25:975-978. 37. Meria $P$, Milcent $S$, Desgrandchamps $F$ et al: Management of pelvic stones larger than $20 \mathrm{~mm}$ : laparoscopic transperitoneal pyelolithotomy or percutaneous nephrolithotomy? Urol Int 2005;75: 322-326.

38. Madi R, Hemal A. Robotic Pyelolithotomy, Extended Pyelolithotomy, Nephrolithotomy, and Anatrophic Nephrolithotomy. J Endourol 2018;32:S73-S81.

39. Favorito LA, Brito DA, Sampaio FJ. Experimental Model of UpperPole Nephrectomy Using Human Tridimensional Endocasts: Analysis of Vascular Injuries. J Endourol 2011;25:113-118.

40. Sampaio FJ, Pereira-Sampaio MA, Favorito LA. The Pig Kidney as an Endourologic Model: Anatomic Contribution. J Endourol 1998;12:45-50. 41. Pereira-Sampaio MA, Favorito LA, Sampaio FJ. Pig Kidney: Anatomical Relationships Between the Intrarenal Arteries and the Kidney Collecting System. Applied Study for Urological Research and Surgical Training. J Urol 2004;172:2077-2081.

42. Sampaio FJB. Relações anatômicas entre as artérias intra-renais e o sistema coletor do rim. In: Sampaio FJB, eds. Sampaio's Anatomia renal para urologia, Rio de Janeiro: Prensa, 2007:41-52.

43. Pereira-Sampaio MA, Favorito LA, Henry RW, Sampaio FJ. Proportional analysis of pig kidney arterial segments: differences from the human kidney. J Endourol 2007;21:784-788.

44. Pereira-Sampaio MA, Henry RW, Favorito LA, Sampaio FJB. Cranial Pole Nephrectomy in the Pig Model: Anatomic Analysis of Arterial Injuries in Tridimensional Endocasts. J Endourol 2012;26:716-721.

45. Marques-Sampaio BP, Pereira-Sampaio MA, Henry RW, Favorito LA, Sampaio FJ. Dog kidney: Anatomical relationships between intrarenal arteries and kidney collecting system. Anat Rec 2007;290:1017-1022. 46. Shalgum A, Marques-Sampaio BP, Dafalla A, Pereira-Sampaio MA. Anatomical relationship between the collecting system and the intrarenal arteries in the rabbit: contribution for an experimental model. Anat Histol Embryol. 2012;41:130-138.

47. Buys-Gonçalves GF, De Souza DB, Sampaio FJB, Pereira-Sampaio MA. Anatomical relationship between the kidney collecting system and the intrarenal arteries in the sheep: Contribution for a new urological model. Anat Rec 2016;299:405-411.

48. Yoldas A, Dayan MO. Morphological characteristics of renal artery and kidney in rats. Sci World J 2014; article ID 468982.

49. Szymanski J, Olewnik L, Wysiadecki G, Przygocka A, Polguj M, Topol $M$. Proposal for a new classification of the renal artery in the bovine kidney. Veterinarni Medicina 2018;63:63-72.

50. Sampaio FJB. Anatomia intra-renal para cirurgia conservadora de néfrons - Nefrectomia parcial. In: Sampaio FJB, eds. Sampaio's Anatomia renal para urologia, Rio de Janeiro: Prensa, 2007:87-101. 
Received: September 10, 2020

Accepted: November 22, 2020
Corresponding author

Marco Aurélio Pereira-Sampaio

Email: pereira.sampaio@gmail.com 